\title{
Clinical feasibility of 3D-QALAS - Single breath-hold 3D myocardial T1 and T2-mapping
}

Sofia Kvernby, Marcel Jan Bertus Warntjes, Jan Engvall, Carljohan Carlhäll and Tino Ebbers

Journal Article

Tweet

N.B.: When citing this work, cite the original article.

Original Publication:

Sofia Kvernby, Marcel Jan Bertus Warntjes, Jan Engvall, Carljohan Carlhäll and Tino Ebbers, Clinical feasibility of 3D-QALAS - Single breath-hold 3D myocardial T1 and T2-mapping, Magnetic Resonance Imaging, 2017. 38, pp.13-20.

http://dx.doi.org/10.1016/j.mri.2016.12.014

Copyright: Elsevier

http://www.elsevier.com/

Postprint available at: Linköping University Electronic Press

$\underline{\text { http://urn.kb.se/resolve?urn=urn:nbn:se:liu:diva-136856 }}$

(cc) 8 S 8

I.U UNNGDESGS 
Clinical feasibility of 3D-QALAS - single breath-hold 3D myocardial T1- and T2-mapping

Sofia Kvernby MSc1, 2, Marcel Warntjes PhD1, 2, 3, Jan Engvall MD1, 2, 4, Carl-Johan Carlhäll MD 1, 2, 4 and Tino Ebbers $\mathrm{PhD}^{1,2,5, \S}$

1Division of Cardiovascular Medicine, Department of Medical and Health Sciences, Linköping University, Linköping, Sweden

${ }^{2}$ Center for Medical Image Science and Visualization (CMIV), Linköping University, Linköping, Sweden

${ }^{3}$ SyntheticMR AB, Linköping, Sweden

4Department of Clinical Physiology, County Council of Östergötland, Linköping,

Sweden

5Division of Media and Information Technology, Department of Science and Technology, Linköping University, Linköping, Sweden

sCorresponding author

Email addresses:

SK: sofia.kvernby@liu.se

MW: marcel.jan.bertus.warntjes@liu.se

JE: jan.engvall@regionostergotland.se

CJC: carljohan.carlhall@liu.se

TE: tino.ebbers@liu.se

Linköping University, Institutionen för medicin och hälsa, 58183 Linköping, Sweden

Short title: Clinical feasibility of 3D-QALAS 


\section{Abstract}

\section{Purpose}

To investigate the in-vivo precision and clinical feasibility of 3D-QALAS - a novel method for simultaneous three-dimensional myocardial T1- and T2-mapping.

\section{Methods}

Ten healthy subjects and 23 patients with different cardiac pathologies underwent cardiovascular 3T MRI examinations including 3D-QALAS, MOLLI and T2-GraSE acquisitions. Precision was investigated in the healthy subjects between independent scans, between dependent scans and as standard deviation of consecutive scans. Clinical feasibility of 3D-QALAS was investigated for native and contrast enhanced myocardium in patients. Data were analyzed using mean value and 95\% confidence interval, Pearson correlation, Paired t-tests, intraclass correlation and Bland-Altman analysis.

\section{Results}

Average myocardial relaxation time values and SD from eight repeated acquisitions within the group of healthy subjects were $1178 \pm 18.5 \mathrm{~ms}(1.6 \%)$ for T1 with $3 \mathrm{D}$ QALAS, $52.7 \pm 1.2 \mathrm{~ms}(2.3 \%)$ for T2 with 3D-QALAS, $1145 \pm 10.0 \mathrm{~ms}(0.9 \%)$ for T1 with MOLLI and $49.2 \pm 0.8 \mathrm{~ms}(1.6 \%)$ for T2 with GraSE.

Myocardial T1 and T2 relaxation times obtained with 3D-QALAS correlated very well with reference methods; MOLLI for T1 $(r=0.994)$ and T2-GraSE for T2 $(r=0.818)$ in the 23 patients. Average native/post contrast myocardial T1 values from the patients were $1166.2 \mathrm{~ms} / 411.8 \mathrm{~ms}$ for 3D-QALAS and $1174.4 \mathrm{~ms} / 438.9 \mathrm{~ms}$ for MOLLI. Average native myocardial T2 values from the patients were $53.2 \mathrm{~ms}$ for 3DQALAS and 54.4ms for T2-GraSE.

\section{Conclusions}

Repeated independent and dependent scans together with the intra-scan repeatability, demonstrated all a very good precision for the 3D-QALAS method in 
healthy volunteers. This study shows that 3D T1 and T2 mapping in the left ventricle is feasible in one breath hold for patients with different cardiac pathologies using 3D-QALAS.

\section{Keywords}

T1 mapping, T2 mapping, Myocardium, Precision, 3D-QALAS, Validation

\section{Introduction}

Quantification of myocardial relaxation times with cardiovascular magnetic resonance (CMR) provides important information for the non-invasive assessment of myocardial tissue characterization. Diffuse tissue changes have now become possible to assess non-invasively with information from the longitudinal relaxation time (T1) and the transverse relaxation time (T2) by using T1- and T2 mapping methods. Mapping of the longitudinal relaxation time has for example been used in the assessment of acute- and chronic myocardial infarction[1] and in the assessment of diffuse fibrosis in patients with cardiomyopathy[2]. Mapping of the transverse relaxation time can be used in the detection of edematous areas in the myocardium[3] and has for example shown improved detection in patients with acute inflammatory cardiomyopathy[4]. Especially for investigating diffuse tissue changes a good 3D coverage of the complete LV is preferred.

Various methods for myocardial relaxation times mapping are available today that typically provide one $2 \mathrm{D}$ slice of the heart with pixel-wise information about either longitudinal $\mathrm{T} 1$ relaxation time or transverse $\mathrm{T} 2$ relaxation time in one breath hold [5-9]. Coverage of the complete LV using these techniques is time consuming, and the large number of breath-holds can be exhaustive to the patient. Some three- 
dimensional mapping methods for either T1 or T2 quantification do exist, which often are free-breathing or navigator based methods[10-12]. Mapping methods providing simultaneously quantification of both $\mathrm{T} 1$ and $\mathrm{T} 2$ are few but there are some 2D methods, for example by Blume et al[13] and Akçakaya et al[14]. While several three-dimensional (3D) methods for simultaneous $\mathrm{T} 1$ and T2 quantification exist for non-cardiac applications[15-17], so far only one 3D method for cardiac application has been proposed; 3D-QALAS (3D-quantification using an interleaved Look-Locker acquisition sequence with T2 preparation pulse)[18]. Extending upon the recently published consensus document for myocardial T1 mapping [19], which recommends that $\mathrm{T} 1$ quantification is performed on a global or segmental basis, the 3D-QALAS method aims to precisely perform relaxation times mapping of T1 and T2 in all 16 segments of the left ventricular myocardium in a single breath-hold only. The 3D-QALAS method is based on a cardiac triggered 3D spoiled Turbo Field Echo sequence using inversion recovery with an interleaved T2 preparation pulse. The estimation of the relaxation times is based on a simulation of the magnetization vector over the acquisition scheme using an iterative analysis approach, instead of using a conventional curve-fitting algorithm as commonly used in the 2D mapping techniques. The ability to perform 3D T1-/T2-mapping in a single breath hold is advantageous to both 2D mapping and 3D-navigator based mapping with respect to number of breath holds and total acquisition time, which is of importance for the clinical feasibility of a method.

According to the consensus statement for myocardial T1 mapping, accuracy and precision of new pulse sequences should be tested systematically. Several of the existing 2D mapping methods have been validated separately as well as against each other with respect to accuracy and precision[20-22]. Measurements have been performed both in-vivo and in-vitro to estimate the advantages and limitations of 
the different methods. Validation of the 3D-QALAS method has been performed invitro using simulated arrhythmia, various flip angles and a wide range of heart rates. The results showed no effect of these parameters within a clinically relevant range and the method revealed a very good correlation against relaxation times measurements with reference methods, Inversion Recovery for T1 measurement and Multi Echo for T2 measurement. The combined three-dimensional T1- and T2mapping method was also tested in-vivo in 10 healthy volunteers with very good correlation to existing in-vivo 2D mapping methods [18]. These results about the accuracy of T1- and T2-mapping using 3D-QALAS are promising, but for clinical practice and longitudinal follow of patients good robustness and precision of the measurements are also crucial. The usability of quantitative methods is thus dependent on a good precision. Another important factor for the clinical feasibility of the method is the ability to measure not only native T1 and T2, but also postcontrast T1. Finally, the technique should be feasible in patients, for which longer breath holds can be challenging. As the T1 mapping consensus document[19] recommends that T1 quantification is performed on a global or segmental basis, precision and clinical feasibility should also be investigated on a global or segmental basis.

The aim of this study was to investigate the in-vivo precision and clinical feasibility of 3D-QALAS. The precision of the method was investigated through the assessment of native $\mathrm{T} 1$ and $\mathrm{T} 2$ repeatability of independent scans, dependent scans and consecutive scans in healthy volunteers. Clinical feasibility was investigated through the assessment of native $\mathrm{T} 1$ and $\mathrm{T} 2$ together with post-contrast $\mathrm{T} 1 \mathrm{in}$ patients with different cardiac pathologies using 3D-QALAS, MOLLI and T2-GraSE. 


\section{Materials and methods}

All subjects gave written informed consent to participate in the study and approval was granted from the Regional Ethical Review Board in Linköping.

\section{Study populations and CMR protocols}

The study population consisted of ten healthy volunteers and 23 patients. The ten healthy volunteers had no history of cardiovascular disease and underwent four scan blocks during the same day, see table 1 .

Table 1. CMR protocols overview of the four scans performed on the healthy subjects.

\begin{tabular}{lcccc}
\hline Mapping method & $\begin{array}{c}\text { Scan } \\
\text { block 1 }\end{array}$ & $\begin{array}{c}\text { Scan } \\
\text { block 2 }\end{array}$ & $\begin{array}{c}\text { Scan } \\
\text { block 3 }\end{array}$ & $\begin{array}{c}\text { Scan } \\
\text { block 4 }\end{array}$ \\
\hline 3D-QALAS & x 1 & x 1 & x 1 & x 8 \\
\hline MOLLI (3(3)-3(3)-5) & & & & \\
-Apical & x 1 & x 1 & x 1 & \\
-Mid-ventricular & x 1 & x 1 & x 1 & x 8 \\
-Basal & x 1 & x 1 & x 1 & \\
T2-GraSE & & & & \\
-Apical & x 1 & x 1 & x 1 & \\
-Mid-ventricular & x 1 & x 1 & x 1 & x 8 \\
-Basal & x 1 & x 1 & x 1 & \\
\hline
\end{tabular}

The subject was removed from the bore and repositioned between the first and the second scan block to achieve independent measurements and thus investigate repeatability of the method, which is a measure of precision under repeatability conditions according to ISO [23]. The scan protocol for the first, second and third scan blocks consist of three MOLLI acquisitions (apical, mid-ventricular and basal), three T2-GraSE acquisitions (apical, mid-ventricular and basal) and one 3D-QALAS acquisition. The scan protocol for the fourth scan block consists of eight mid- 
ventricular MOLLI acquisitions, eight mid-ventricular T2-GraSE acquisitions and eight 3D-QALAS acquisitions with the aim to investigate precision by using standard deviations of repeated measurements.

A total of 23 patients with clinically significant aortic stenosis (5 patients of which 2 women, average age 66 years), ischemic cardiomyopathy (8 patients of which 3 women, average age 72 years), idiopathic dilated cardiomyopathy ( 7 patients of which 2 women, average age 65 years) or diastolic heart failure ( 3 patients of which 3 women, average age 79 years) were consecutively included in the study. The patients underwent a MR scanning protocol including injection of $0.2 \mathrm{ml} / \mathrm{kg}$ Gadovist-contrast agent. Pre-contrast acquisition included one 3D-QALAS, one midventricular MOLLI and one mid-ventricular T2-GraSE acquisition. Post contrast, one 3D-QALAS and one mid-ventricular MOLLI acquisition were performed.

\section{MR sequence parameters}

All CMR scans were performed using a 3T system, Philips Ingenia (Best, The Netherlands).

The 3D-QALAS acquisition had a resolution of $2.0 \mathrm{~mm} \times 2.0 \mathrm{~mm}$ in plane and a slice thickness of $12.0 \mathrm{~mm}$ (reconstructed to $2.0 \mathrm{~mm} \times 2.0 \mathrm{~mm} \times 6.0 \mathrm{~mm}$ ), flip angle of $5^{\circ}$, SENSE factor of 2 in phase direction and 1.2 in slice direction, repetition time was $2.6 \mathrm{~ms}$ and echo time was $1.2 \mathrm{~ms}$. The 3D-QALAS acquisition was performed during a breath hold of 15 heartbeats and provides 13 slices of the left ventricular myocardium. The MOLLI acquisitions were performed using 3(3)3(3)5 acquisitions, which has recently demonstrated the best precision among several T1 mapping methods[20]. The resolution was $1.22 \mathrm{~mm} \times 1.22 \mathrm{~mm}$ in plane and with a slice thickness of $10 \mathrm{~mm}$. A flip angle of $35^{\circ}$ was used together with a repetition time of 
$2.4 \mathrm{~ms}$ and an echo time of $1.13 \mathrm{~ms}$. The MOLLI acquisition was performed during a breath hold of 17 heartbeats.

The T2-GraSE acquisition had the same resolution as the MOLLI acquisition and was performed during a breath hold of 15 heartbeats. Nine different echo times, range between $9 \mathrm{~ms}$ and $84 \mathrm{~ms}$, were used for the calculation of the T2-map and the repetition time was $1000 \mathrm{~ms}$.

\section{Image analysis}

Generation of the 3D-QALAS T1- and T2-maps was performed on a standalone version of SyMRI ${ }^{\circledR}$ (SyntheticMR, Sweden). 3D-QALAS provides 5 data points for each voxel, 1 after a T2prep pulse and 4 after an inversion pulse, all acquired at late diastole [18]. An iterative approach was applied to calculate the steady-state magnetization vector under the influence of the T2prep pulse, the inversion pulse, the continuous RF excitation and spoiling during the acquisition together with the delay times in between the acquisitions. Iterations were driven by a cost-function to minimize the difference between the simulated magnetization vector and the measured signal intensity of the 5 data points. T1- and T2-maps from the reference methods were generated directly on the MRI scanner console. The generated T1and T2-maps from all methods were analyzed using Segment v 1.9 R3644 (http://segment.heiberg.se) [24]. For the three first scans of the healthy volunteers, myocardial data were manually contoured with respect to epi- and endo-cardial borders. Based on these manual contours, 17-segment bull's eye plots were created automatically by the analysis software. For the fourth scan, only mid-ventricular data were acquired for the references and they were thus only analyzed as six midventricular segments, according to the 17 -segment model recommended by the American Heart Association (AHA)[25]. Since the 3D-QALAS acquisition from the fourth scan provides 13 slices, a bull's eye plot was created as a first step but for 
comparison with the reference methods only the data from the six mid-ventricular segments of the bull's eye plot were included in the results.

Patient data were also contoured and analyzed in Segment. The mid-ventricular data from the reference methods were analyzed as six segments. 3D-QALAS patient data were analyzed as a bull's eye plot but for comparison with reference methods, only data from the six mid-ventricular segments were included.

Unless otherwise stated, myocardial $\mathrm{T} 1$ - and $\mathrm{T} 2$ relaxation times data were presented globally as an average over the slice/slices.

\section{Statistical analysis}

For 3D-QALAS, MOLLI and T2 GraSE, relaxation times measurement variations between the three first scans were calculated as mean difference between scans and the corresponding $95 \%$ confidence interval. Variations between the first and second scan illustrate precision under repeatability conditions and variations between the second and third scan illustrate precision under intra-scan conditions. The agreement between separate measurements, assessment of repeatability, was determined by using the Pearson correlation analysis for scan 1 versus scan 2 and scan 2 versus scan 3 in the healthy volunteers.

The eight repeated examinations from scan block four were analyzed by using intrascan standard deviation. Paired t-tests were conducted to examine whether there are significant differences between the eight acquisitions in their sequential order. Mean value and standard deviation were calculated for the subjects. An intraclass correlation analysis using a two-way random model was performed on the data from the fourth scan to get a measure of how strongly subjects in the same group resemble each other, in this case the individual groups are represented by the eight repeated acquisitions with the different mapping methods. A Bland-Altman analysis 
was performed for the patient data where bias and limits of agreement were calculated. The correlation between patient data obtained with 3D-QALAS and the reference methods were analyzed using the Pearson correlation.

\section{Results}

Ten healthy volunteers and 23 patients with different cardiac pathologies were included in this study. A characteristics summary of the subjects can be seen in table 2.

Table 2. Characteristics of the subjects included in the study.

\begin{tabular}{|c|c|c|c|c|c|c|}
\hline Category & $\begin{array}{c}\text { Number of } \\
\text { subjects }\end{array}$ & Gender & $\begin{array}{c}\text { Age } \\
\text { (years } \pm \text { sd) }\end{array}$ & $\begin{array}{c}\text { Height } \\
\text { (cm } \pm \text { sd) }\end{array}$ & $\begin{array}{l}\text { Weight } \\
\text { (kg士sd) }\end{array}$ & $\begin{array}{c}\text { Heart rate } \\
\text { (bpm } \pm \text { sd) }\end{array}$ \\
\hline Healthy & 10 & $\begin{array}{l}4 \text { Female, } \\
6 \text { Male }\end{array}$ & $38 \pm 7$ & $178 \pm 7$ & $71 \pm 11$ & $58 \pm 10$ \\
\hline Aortic stenosis & 5 & $\begin{array}{l}2 \text { female, } \\
3 \text { Male }\end{array}$ & $66 \pm 4$ & $171 \pm 10$ & $88 \pm 17$ & $70 \pm 6$ \\
\hline $\begin{array}{c}\text { Ischemic } \\
\text { cardiomyopathy }\end{array}$ & 8 & $\begin{array}{l}3 \text { Female, } \\
8 \text { Male }\end{array}$ & $72 \pm 6$ & $174 \pm 10$ & $81 \pm 11$ & $66 \pm 10$ \\
\hline $\begin{array}{c}\text { Idiopathic } \\
\text { dilated } \\
\text { cardiomyopathy }\end{array}$ & 7 & $\begin{array}{l}2 \text { Female, } \\
5 \text { Male }\end{array}$ & $71 \pm 8$ & $174 \pm 7$ & $83 \pm 13$ & $53 \pm 12$ \\
\hline $\begin{array}{l}\text { Diastolic heart } \\
\text { failure }\end{array}$ & 3 & 3 Female & $79 \pm 8$ & $158 \pm 4$ & $72 \pm 11$ & $52 \pm 7$ \\
\hline
\end{tabular}

\section{Healthy volunteers}

All data were successfully acquired for the healthy volunteers.

Myocardial relaxation times from the two independent scans, first scan versus the second scan, showed a less good correlation than myocardial relaxation times from the second scan versus the third scan, for both 3D-QALAS and the reference methods. The correlation coefficients are presented in table 3 together with the 
average difference and $95 \%$ confidence interval (CI) between the three different scans, where the largest $95 \% \mathrm{CI}$ of the difference is found between the first two independent scans.

Table 3. Comparative result from the first three scans, based on ten healthy volunteers. Mean difference is expressed as the average difference from all healthy volunteers.

\begin{tabular}{|c|c|c|}
\hline & $\begin{array}{l}\text { Scan } 1 \text { vs. scan } 2 \\
\text { Independent scans }\end{array}$ & $\begin{array}{l}\text { Scan } 2 \text { vs scan } 3 \\
\text { Dependent scans }\end{array}$ \\
\hline \multicolumn{3}{|l|}{ 3D-QALAS (T1) } \\
\hline Pearson correlation & $\mathrm{r}=0.782$ & $r=0.915$ \\
\hline Mean difference , $95 \% \mathrm{CI}$ [ms] & $\Delta=-26.1,(-56.1 ; 3.8)$ & $\Delta=-1.7,(-14.0 ; 10.5)$ \\
\hline \multicolumn{3}{|l|}{ MOLLI } \\
\hline Pearson correlation & $\mathrm{r}=0.583$ & $\mathrm{r}=0.812$ \\
\hline Mean difference, $95 \%$ CI [ms] & $\Delta=-1.6,(-22.7 ; 19.5)$ & $\Delta=-7.0,(-23.3 ; 9.3)$ \\
\hline \multicolumn{3}{|l|}{ 3D-QALAS (T2) } \\
\hline Pearson correlation & $r=0.624$ & $r=0.648$ \\
\hline Mean difference , $95 \%$ CI [ms] & $\Delta=-1.8,(-3.0 ;-0.6)$ & $\Delta=0.1,(-1.3 ; 1.6)$ \\
\hline \multicolumn{3}{|l|}{ GraSE } \\
\hline Pearson correlation & $r=0.588$ & $r=0.903$ \\
\hline Mean difference , $95 \%$ CI [ms] & $\Delta=-1.0,(-2.4 ; 0.4)$ & $\Delta=0.7,(-0.2 ; 1.5)$ \\
\hline
\end{tabular}

Average relaxation time value and standard deviation of all ten subjects, i.e. on a group level, for the four scans are illustrated in figure 1 for T1 and figure 2 for T2 (scan four is illustrated as the eight separate acquisitions, denoted as SD1 - SD8). Results from the fourth scan are presented as average value and standard deviation over the eight repeated acquisitions for each healthy volunteer, i.e. results on an individual level, (figure 3 and 4). The overall average myocardial relaxation time value and standard deviation from eight repeated acquisitions within the population group of healthy subject with 3D-QALAS is $1178 \pm 18.5 \mathrm{~ms}(1.6 \%)$ for T1 measurements and $52.7 \pm 1.2 \mathrm{~ms}(2.3 \%)$ for T2 measurements. The corresponding average value and standard deviations for the reference methods are $1145 \pm 10.0$ 
ms $(0.9 \%)$ for $\mathrm{T} 1$ measurements with MOLLI and $49.2 \pm 0.8 \mathrm{~ms}(1.6 \%)$ for $\mathrm{T} 2$ measurements with GraSE. An example of relaxation times maps from the eight repeated acquisitions are shown in figure 5 .

Paired t-tests of the data from scan four do not show any significant differences ( $p>0.05)$ between the eight acquisitions on a group level in its sequential order, i.e. SD1 vs SD2 and SD2 vs SD3 and so on, for neither 3D-QALAS nor the reference methods. The result from the intraclass correlation analysis shows that both 3DQALAS and the reference methods have a very high reliability according to intraclass correlation coefficient (3D-QALAS T1: 0.873, 3D-QALAS T2: 0.842, MOLLI: 0.974 and GraSE: 0.964).

\section{Patients}

All patients completed the study. Data were successfully acquired in all cases, except from one where only native data could be acquired.

Mid-ventricular T1 and T2 maps from 3D-QALAS and the reference methods for a patient with ischemic cardiomyopathy can be seen in figure 6. Myocardial T1 and T2 relaxation times obtained with 3D-QALAS correlate very well with reference methods, MOLLI for T1 $(r=0.994)$ and T2-GraSE for T2 $(r=0.818)$ in the 23 included patients. Data are shown in Bland-Altman plots, figure 7 and 8, where myocardial relaxation times of the healthy volunteers from the first scan are included. Bias and $\pm 1.96 \mathrm{SD}$ are $-7.3 \mathrm{~ms} \pm 97.9 \mathrm{~ms}$ for $\mathrm{T} 1$ and $0.1 \mathrm{~ms} \pm 8.2 \mathrm{~ms}$ for $\mathrm{T} 2$. Overall average \pm SD native/post contrast myocardial T1 values from the patients are $1166.2 \pm 63.9$ $\mathrm{ms} / 411.8 \pm 64.3 \mathrm{~ms}$ for 3D-QALAS and $1174.4 \pm 55.2 \mathrm{~ms} / 438.9 \pm 52.4 \mathrm{~ms}$ for MOLLI. Overall average \pm SD native myocardial T2 values from the patients are 53.2 $\pm 5.6 \mathrm{~ms}$ for 3D-QALAS and $54.4 \pm 6.9 \mathrm{~ms}$ for T2-GraSE. 


\section{Discussion}

This study investigated the precision of simultaneous native T1 and T2 mapping using 3D-QALAS in healthy volunteers and the feasibility of the method for T1 and T2 quantification of native and post contrast myocardium in patients. Precision has been investigated between two independent scans, between two dependent scans and as standard deviation of eight consecutive scans in ten healthy volunteers. All methods (MOLLI, GraSE and 3D-QALAS) showed good precision. The standard deviation of eight consecutive scans was slightly better using MOLLI for T1 and GraSE for T2 than for 3D-QALAS, while the correlation for two independent scans was slightly better using 3D-QALAS. Myocardial T1 and T2 relaxation times obtained with 3D-QALAS correlated very well with reference methods, MOLLI and GraSE, in the 23 included patients for both native as well as post contrast myocardial tissue.

Eight repeated measurements were performed to estimate precision through intrascan standard deviation of dependent acquisitions. The overall average standard deviations were slightly larger with 3D-QALAS for both T1- and T2 measurements than with the reference methods, MOLLI for T1- and GraSE for T2 measurements. The intra-scan standard deviation from eight acquisitions was $1.6 \%$ with 3DQALAS T1, $0.9 \%$ with MOLLI, $2.3 \%$ with 3D-QALAS T2 and $1.6 \%$ with GraSE. Relaxation times values representing healthy myocardial tissue are well-known to differ depending on for example vendor and mapping method used, which is a problem when comparing values with each other [20], as in this study where myocardial relaxation times for the consecutive scans estimated with the 3D-QALAS method are slightly longer than with the reference methods. Using intraclass correlation analysis, individual differences of the methods could be taken into account by separating the subjects from each other in the analysis. Intraclass 
correlation analysis of the eight scans indicated very good repeatability of 3DQALAS for T1 and T2 mapping, and even better for MOLLI and T2-GraSE.

However, the larger $95 \%$ confidence interval between the independent than between the dependent scans of all methods indicates that the precision cannot be completely assessed by the consecutive scans. For example the positioning of the patient, positioning of the shim-box and the exact slice positioning are factors that seem to influence the outcome of myocardial relaxation times mapping for both the 3D-QALAS method and for the reference methods. The evaluation using independent scans is therefore of major importance, as it best reflects the expected precision in healthy subjects as well as in patients. 3D-QALAS showed a very good correlation between both the independent scans, scan block 1 and scan block 2, and the dependent scans, scan block 2 and scan block 3. This indicates that 3DQALAS is a robust method even when effects connected to independent scan situations arise. The robustness of the method, even between independent scans, might be due to the fact that 3D-QALAS excites a 3D slab instead of just a 2D slab that reduces the influence of slice positioning effects. In addition, the iterative analysis approach handles several possibly confounding factors that could affect the estimate of $\mathrm{T} 1$ and $\mathrm{T} 2$ relaxation times, such as the T2 dependency and the influence of heart rate, making the 3D-QALAS method more stable than methods using conventional curve-fitting approach.

The native T1 times for healthy subjects presented in this study, both with MOLLI and 3D-QALAS, are in the upper part of the normal range reported in earlier studies [26] and also compared to earlier in-vivo results with 3D-QALAS[18]. Healthy myocardial T1 relaxation times seems to differ fairly much between individuals, meaning that the inter subject variability is larger than the intra 
subject variability. Ten healthy subjects are not sufficient to establish a range of normal/healthy myocardial T1 or T2 relaxation times, which also isn't the objective of this study. To obtain reference values of normal/healthy myocardial T1 or T2 relaxation times, the study population has to be substantially expanded and a multi-center study might be of interest.

As described in earlier work[18], performing relaxation times mapping in a whole $3 \mathrm{D}$-volume instead of in a $2 \mathrm{D}$ slice, requires sampling of larger quantities of data. To be able to sample the larger amount of data without losing valuable information, such as the important in-plane resolution or veracity in the relaxation time measurement, some other considerations has to be made regarding for example a slightly longer acquisition window, $<230 \mathrm{~ms}$, and a larger slice thickness, $12 \mathrm{~mm}$, than the conventional 2D mapping methods. These compromises have to be compared to the advantage of getting 3D data from both $\mathrm{T} 1$ and $\mathrm{T} 2$ in one breath hold.

The standard deviations presented for the patient data in this study are much larger than for the healthy population data, which is reasonable since the patients suffer from different cardiac pathologies that are well known to affect the myocardial relaxation times. Moreover, the standard deviation for patients are based on relaxation times from 23 different subjects and one measurement per subject and method while the standard deviations presented for healthy volunteers are based on 8 consecutive measurements within the same subject and should thus not be compared.

A very good correlation is also seen between the 3D-QALAS method and the reference methods for patients with different cardiac pathologies. These patients 
were investigated with the same 3D-QALAS scan before and after injection of gadolinium based contrast agent (figure 7 and 8), indicating that 3D-QALAS has great robustness in a large range of myocardial relaxation times.

The aim to investigate clinical feasibility was performed by including patients with different cardiac pathologies. Performing an MRI examination of cardiac patients might induce various difficulties that not in the same extent need to be considered when examining merely healthy volunteers. Difficulties for patients holding their breath during acquisition, ECG-triggering disturbances due to abnormal electrocardiograms and trouble lying still under examination are example of such difficulties that might affect the result of the relaxation time mapping. We investigated the clinical feasibility of 3D-QALAS to see whether T1 and T2 can be feasibly measured with 3D-QALAS in patients where difficulties, as stated above, occur. If 3D-QALAS can be used to discriminate different pathologies with respect to relaxation times, is another question that must be a topic of future research.

The 3D-QALAS sequence has in this study shown applicability and robustness and provides reliable relaxation time mapping results for both $\mathrm{T} 1$ and $\mathrm{T} 2$.

\section{Limitations}

Increasing the number of independent scans in the study would be desirable to achieve an even more accurate measure of precision, but since it is demanding for the subject (this experimental setup provided in average 62 breath holds per subject) and also an extremely time consuming procedure to perform independent scans, the number of scans were limited. Other limitations are the small number of subjects included in the study and the large age range of the subjects. Since the study included in-vivo examinations, no specific heart rate was used and heart rate 
dependency of a mapping method might therefore influence the outcome. Only midventricular data from the reference methods were used in the repeated consecutive scans, while 3D-QALAS included data from several slices to create comparative mid-ventricular data in the analysis program.

\section{Conclusions}

The results from this study indicate that 3D T1 and T2 mapping in the left ventricle is feasible in one breath hold for patients with different cardiac pathologies. Repeated independent and dependent scans together with the intra-scan repeatability as a measure of precision demonstrated all a very good precision for the 3D-QALAS method in healthy volunteers. Relaxation times obtained with 3DQALAS also showed a good correlation with existing 2D T1 and T2 mapping methods.

\section{Acknowledgements}

The authors acknowledge the help from Johan Kihlberg, Christer Holm, Henrik Ekman, Andreas Bussman, Ingela Ericsson and Jennie Kemppi at CMIV for contributing in acquiring patient data and Urban Alehagen for contributing to recruit patients together with Karl Wahlin for statistical advice. This study was partly funded by the Swedish Heart and Lung Foundation, grants 20120449 and 20140398, and the Swedish Research Council, grant 2014-6191. 


\section{References}

[1] Messroghli DR, Walters K, Plein S, Sparrow P, Friedrich MG, Ridgway JP, et al. Myocardial T1 mapping: application to patients with acute and chronic myocardial infarction. Magnetic Resonance in Medicine 2007;58(1):34-40.

[2] Puntmann VO, Voigt T, Chen Z, Mayr M, Karim R, Rhode K, et al. Native T1 Mapping in Differentiation of Normal Myocardium From Diffuse Disease in Hypertrophic and Dilated Cardiomyopathy. JACC: Cardiovascular Imaging 2013;6(4):475-84.

[3] Giri S, Chung Y-C, Merchant A, Mihai G, Rajagopalan S, Raman S, et al. T2 quantification for improved detection of myocardial edema. Journal of Cardiovascular Magnetic Resonance 2009;11(1):56.

[4] Thavendiranathan P, Walls M, Giri S, Verhaert D, Rajagopalan S, Moore S, et al. Improved Detection of Myocardial Involvement in Acute Inflammatory Cardiomyopathies Using T2 Mapping. Circulation: Cardiovascular Imaging 2012;5(1):102-10.

[5] Messroghli DR, Radjenovic A, Kozerke S, Higgins DM, Sivananthan MU, Ridgway JP. Modified look-locker inversion recovery (MOLLI) for high-resolution T 1 mapping of the heart. Magnetic Resonance in Medicine 2004;52(1):141-6.

[6] Piechnik SK, Ferreira VM, Dall'Armellina E, Cochlin LE, Greiser A, Neubauer S, et al. Shortened Modified Look-Locker Inversion recovery (ShMOLLI) for clinical myocardial T1-mapping at 1.5 and $3 \mathrm{~T}$ within a 9 heartbeat breathhold. Journal of Cardiovascular Magnetic Resonance 2010;12(1).

[7] Chow K, Flewitt JA, Green JD, Pagano JJ, Friedrich MG, Thompson RB. Saturation recovery single-shot acquisition (SASHA) for myocardial T1 mapping. Magnetic Resonance in Medicine 2013:n/a-n/a.

[8] Weingartner S, Akcakaya M, Berg S, Kissinger K, Manning W, Nezafat R. Heartrate independent myocardial T1-mapping using combined saturation and inversion preparation pulses. Journal of Cardiovascular Magnetic Resonance 2013;15(Suppl 1):P46.

[9] Huang T-Y, Liu Y-J, Stemmer A, Poncelet BP. T2 measurement of the human myocardium using a T2-prepared transient-state trueFISP sequence. Magnetic Resonance in Medicine 2007;57(5):960-6.

[10] Ding H, Fernandez-de-Manuel L, Schär M, Schuleri KH, Halperin H, He L, et al. Three-dimensional whole-heart T2 mapping at 3T. Magnetic Resonance in Medicine 2015;74(3):803-16.

[11] Coniglio A, Di Renzi P, Vilches Freixas G, Della Longa G, Santarelli A, Capparella R, et al. Multiple 3D inversion recovery imaging for volume T1 mapping of the heart. Magnetic Resonance in Medicine 2013;69(1):163-70.

[12] Weingärtner S, Akçakaya M, Roujol S, Basha T, Stehning C, Kissinger KV, et al. Free-breathing post-contrast three-dimensional T1 mapping: Volumetric assessment of myocardial T1 values. Magnetic Resonance in Medicine 2015;73(1):214-22.

[13] Blume U, Lockie T, Stehning C, Sinclair S, Uribe S, Razavi R, et al. Interleaved T1 and $\mathrm{T} 2$ relaxation time mapping for cardiac applications. Journal of Magnetic Resonance Imaging 2009;29(2):480-7.

[14] Akçakaya M, Weingärtner S, Basha TA, Roujol S, Bellm S, Nezafat R. Joint myocardial T1 and T2 mapping using a combination of saturation recovery and T2-preparation. Magnetic Resonance in Medicine 2015:n/a-n/a. 
[15] Deoni SCL, Rutt BK, Peters TM. Rapid combined T1 and T2 mapping using gradient recalled acquisition in the steady state. Magnetic Resonance in Medicine 2003;49(3):515-26.

[16] Warntjes JBM, Dahlqvist O, Lundberg P. Novel method for rapid, simultaneous T1, $\mathrm{T}^{*} 2$, and proton density quantification. Magnetic Resonance in Medicine 2007;57(3):528-37.

[17] Deoni SCL, Peters TM, Rutt BK. High-resolution T1 and T2 mapping of the brain in a clinically acceptable time with DESPOT1 and DESPOT2. Magnetic Resonance in Medicine 2005(1):237.

[18] Kvernby S, Warntjes MJB, Haraldsson H, Carlhäll C-J, Engvall J, Ebbers T. Simultaneous three-dimensional myocardial T1 and T2 mapping in one breath hold with 3D-QALAS. Journal of Cardiovascular Magnetic Resonance 2014;16(1):1-14.

[19] Moon JC, Messroghli DR, Kellman P, Piechnik SK, Robson MD, Ugander M, et al. Myocardial T1 mapping and extracellular volume quantification: a Society for Cardiovascular Magnetic Resonance (SCMR) and CMR Working Group of the European Society of Cardiology consensus statement. J Cardiovasc Magn Reson 2013;15(1):92.

[20] Kellman P, Hansen MS. T1-mapping in the heart: accuracy and precision. Journal of Cardiovascular Magnetic Resonance 2014;16(1):2.

[21] Messroghli DR, Plein S, Jones TR, Sivananthan MU, Higgins DM, Ridgway JP, et al. Human myocardium: Single-breath-hold MR T1 mapping with high spatial resolution - Reproducibility study. Radiology 2006;238(3):1004-12.

[22] Roujol S, Weingartner S, Foppa M, Chow K, Kawaji K, Kissinger KV, et al. Accuracy and reproducibility of four T1 mapping sequences: a head-to-head comparison of MOLLI, ShMOLLI, SASHA, and SAPPHIRE. Journal of Cardiovascular Magnetic Resonance 2014;16(Suppl 1):026.

[23] Standardization IOf. International Organization for Standardization ISO/TC 69/SC 6. 1994.

[24] Heiberg E, Sjogren J, Ugander M, Carlsson M, Engblom H, Arheden H. Design and validation of Segment - freely available software for cardiovascular image analysis. BMC Medical Imaging 2010;10(1):1.

[25] Cerqueira MD, Weissman NJ, Dilsizian V, Jacobs AK, Kaul S, Laskey WK, et al. Standardized myocardial segmentation and nomenclature for tomographic imaging of the heart a statement for healthcare professionals from the cardiac imaging committee of the Council on Clinical Cardiology of the American Heart Association. Circulation 2002;105(4):539-42.

[26] von Knobelsdorff-Brenkenhoff F, Prothmann M, Dieringer M, Wassmuth R, Greiser A, Schwenke C, et al. Myocardial T1 and T2 mapping at 3 T: reference values, influencing factors and implications. Journal of Cardiovascular Magnetic Resonance 2013;15(1):53. 
Figure captions

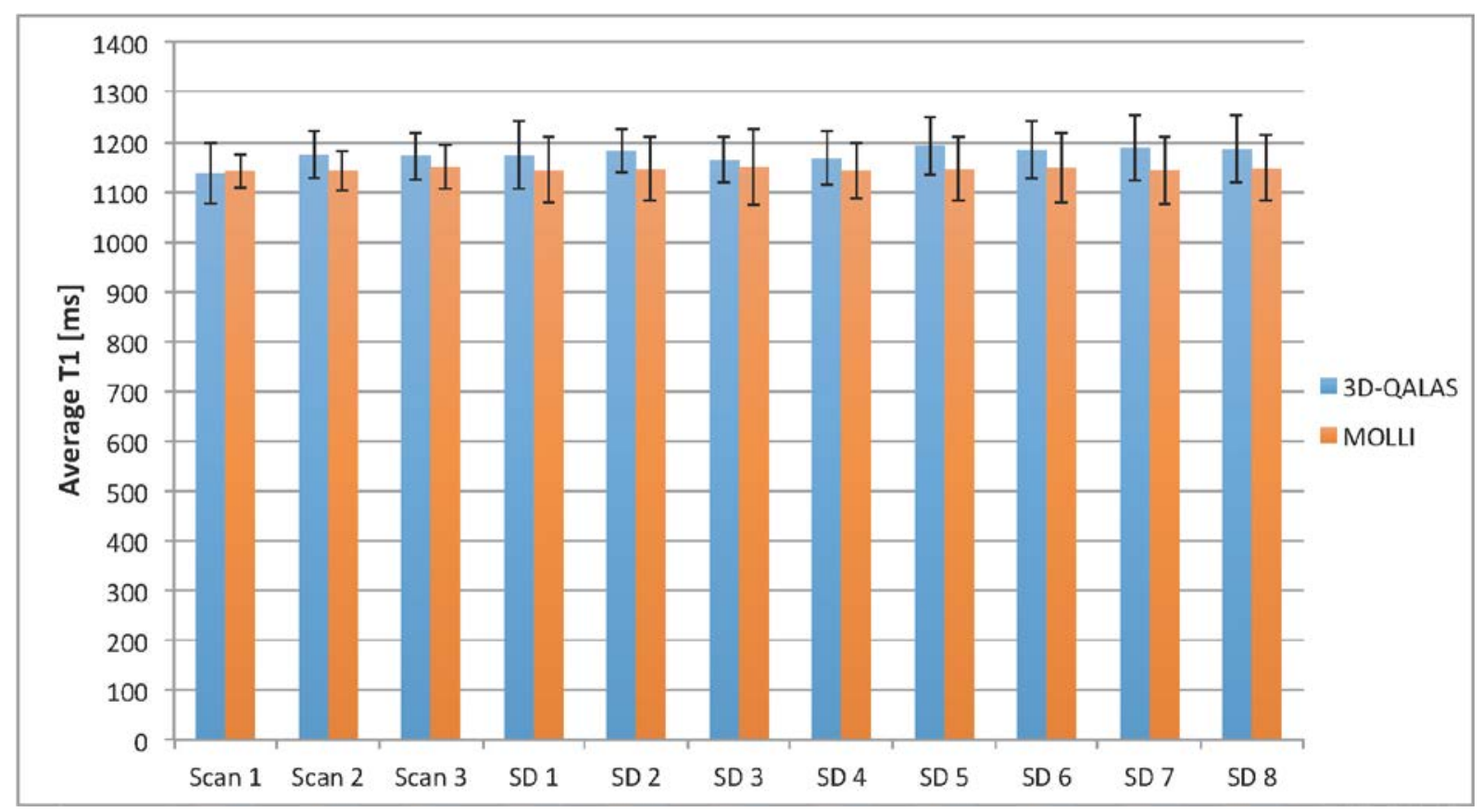

Figure 1. Average myocardial T1 values and standard deviations from the group of ten healthy volunteers for each scan (scan four is illustrated as the eight separate acquisitions, denoted as SD1 - SD8). T1 values with 3D-QALAS and MOLLI are represented.

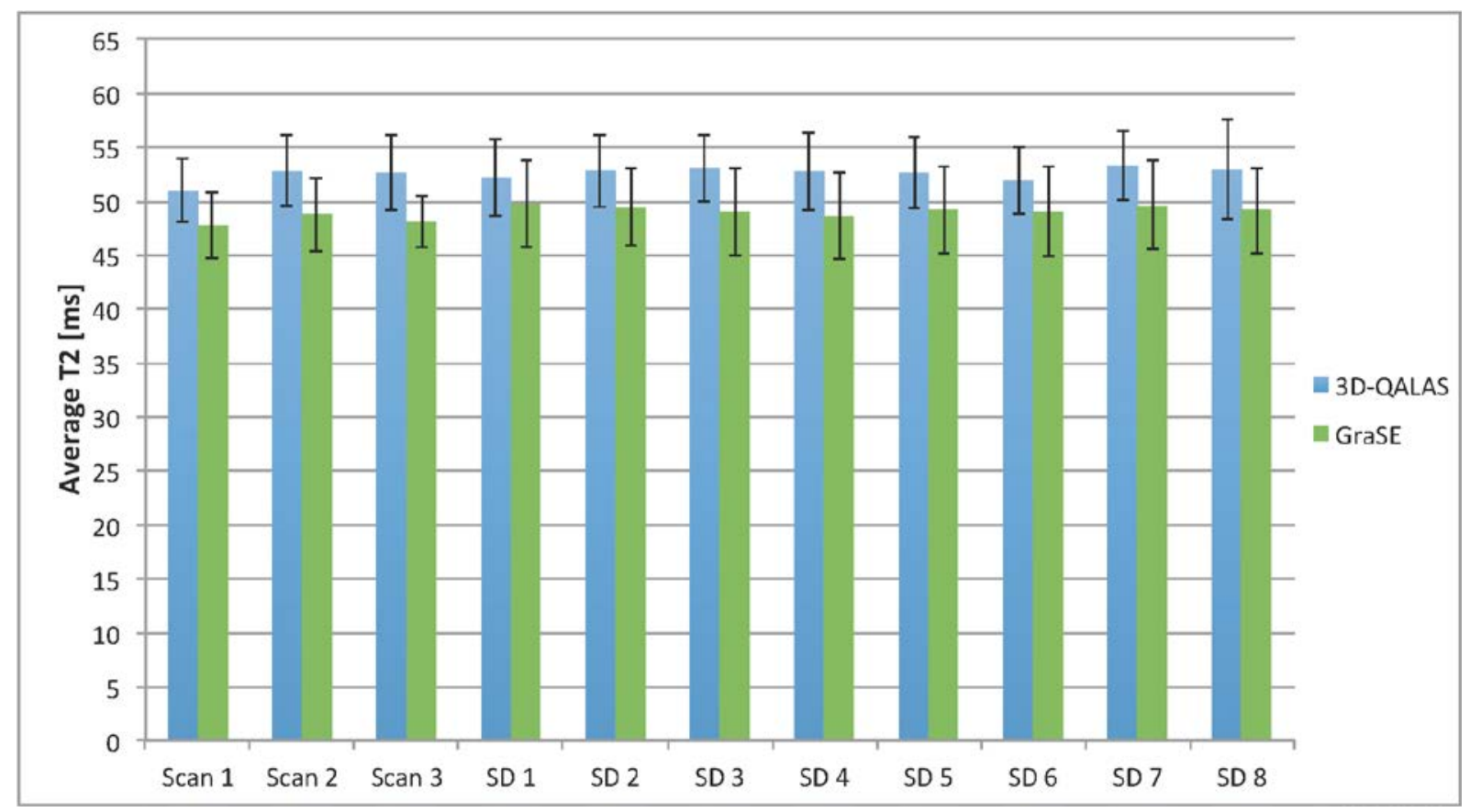


Figure 2. Average myocardial T2 values and standard deviations from the group of ten healthy volunteers for each scan. T2 values with 3D-QALAS and GraSE are represented.

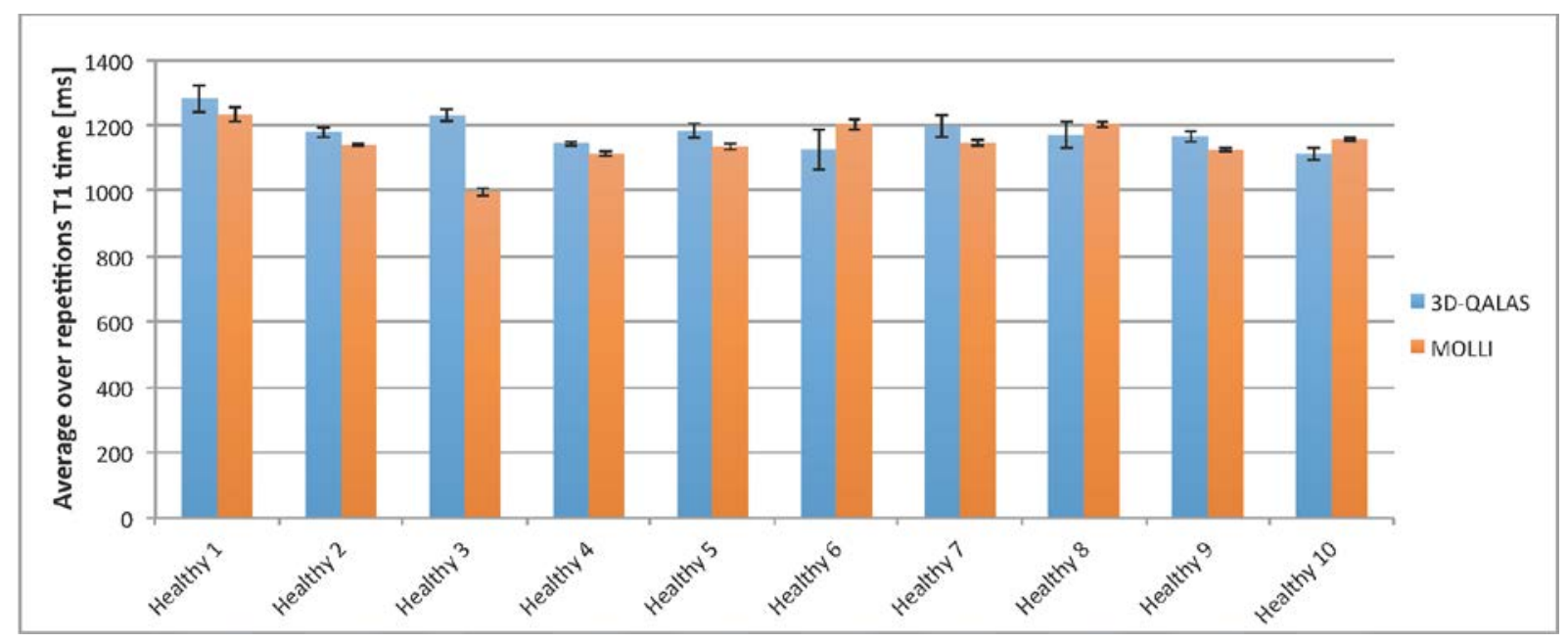

Figure 3. Individual intra scan-repeatability of 3D-QALAS and MOLLI in ten healthy subjects. Data from each subject are represented by average value and standard deviation of the eight repeated acquisitions from scan 4.

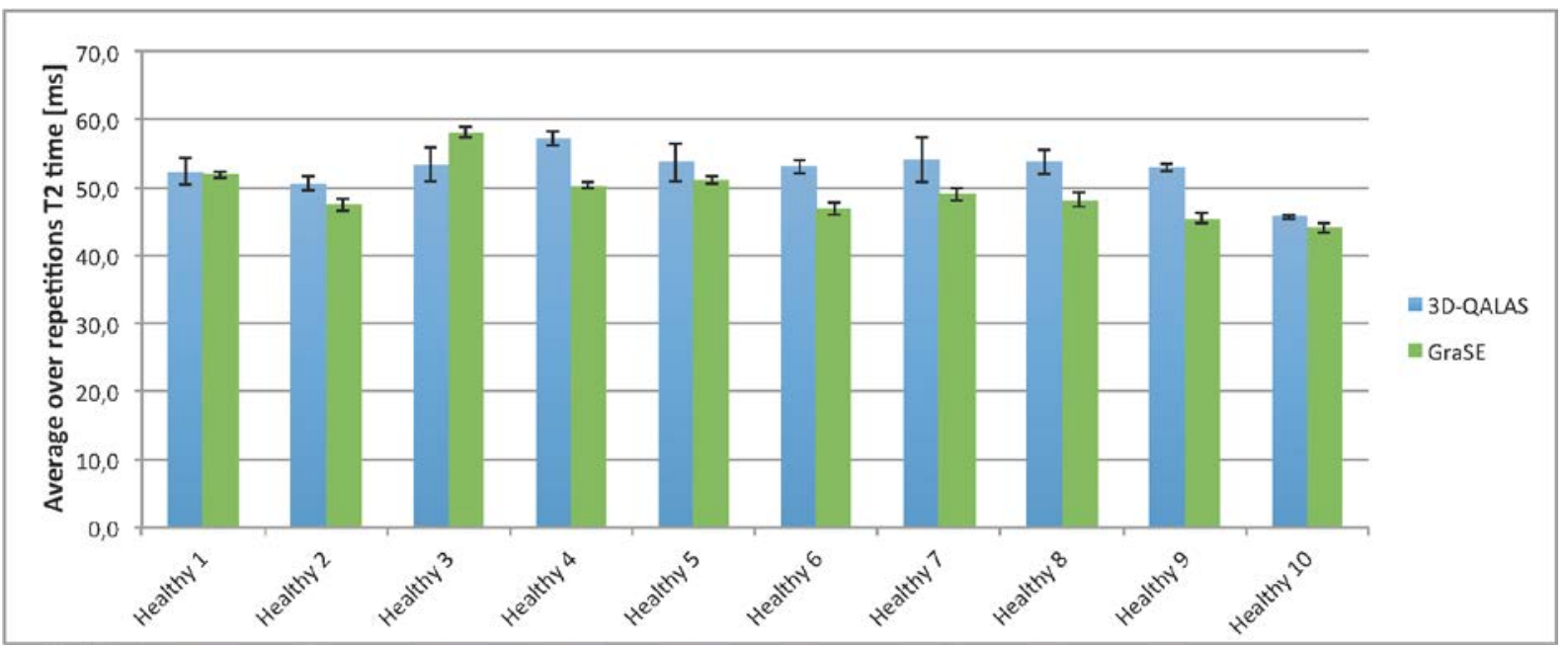

Figure 4. Individual intra scan-repeatability of 3D-QALAS and T2-GraSE in ten healthy subjects. Data from each subject are represented by average value and standard deviation of the eight repeated acquisitions from scan 4. 


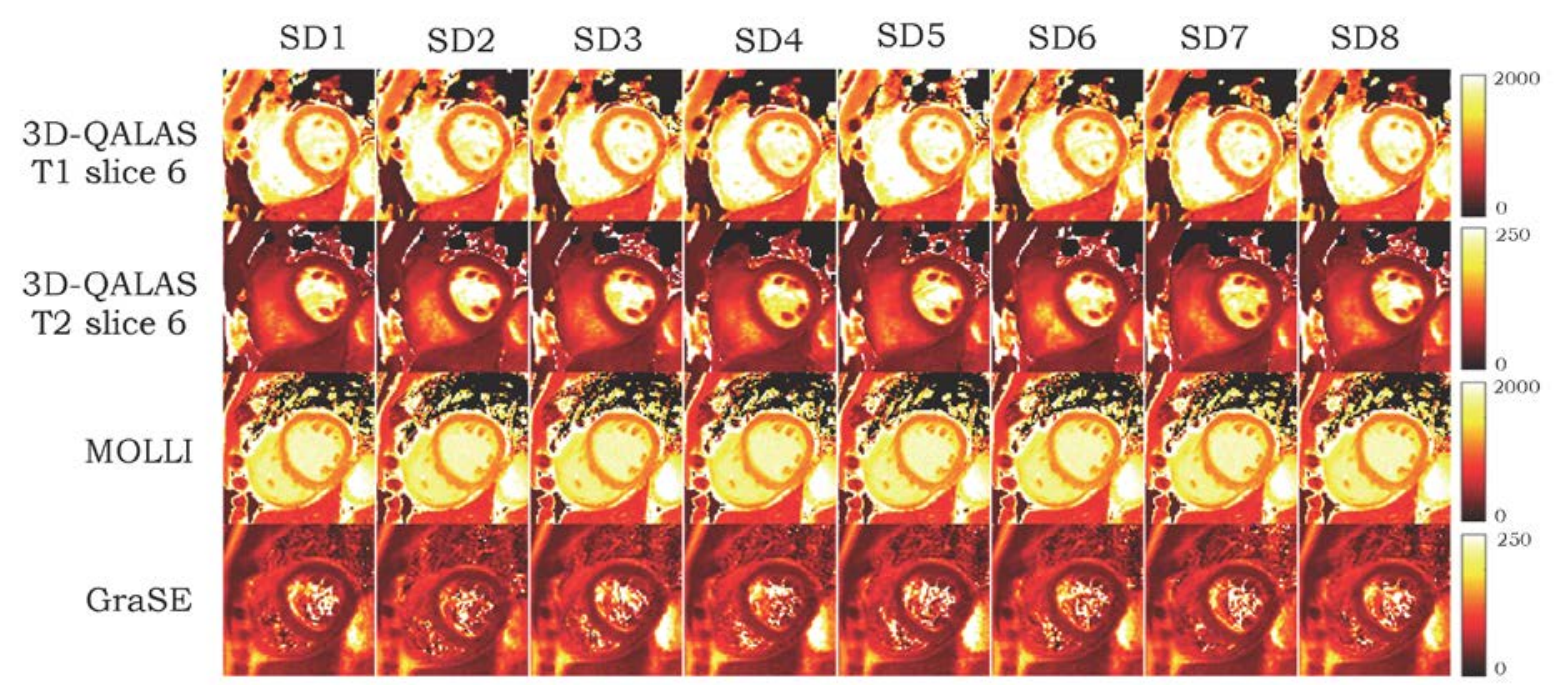

Figure 5. Example of relaxation times maps from the eight repeated acquisitions (SD1-SD8) of a healthy volunteer. Image shows slice 6 with 3D-QALAS for both T1 and T2 and a mid-ventricular slice with the 2D reference methods.

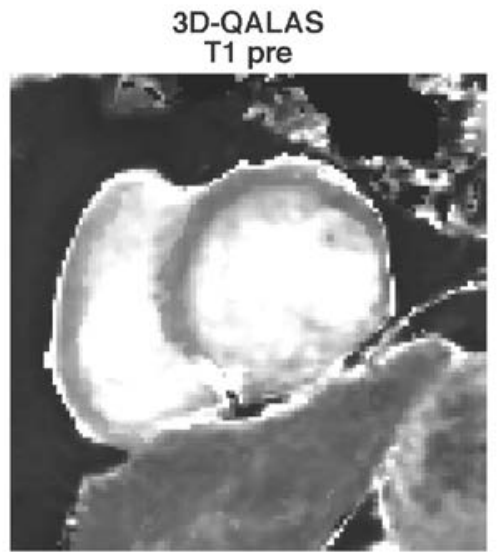

MOLLI pre
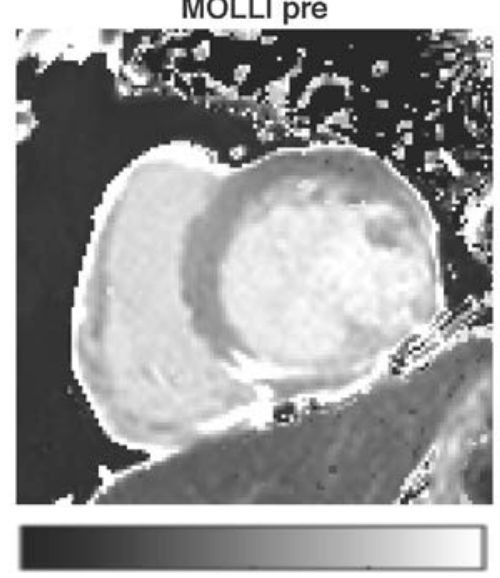

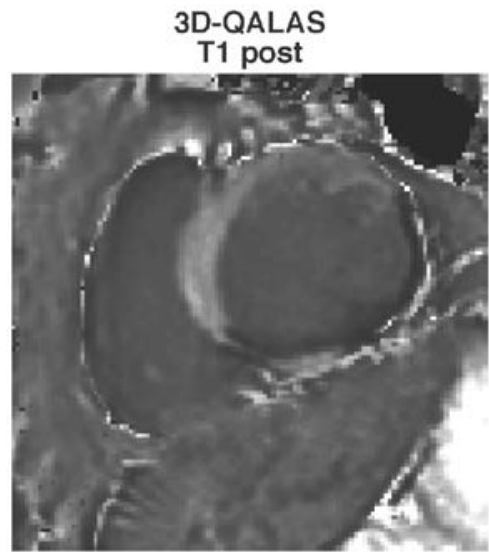

MOLLI post

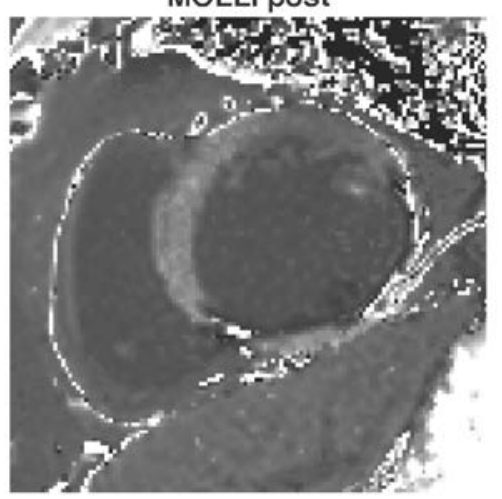

500

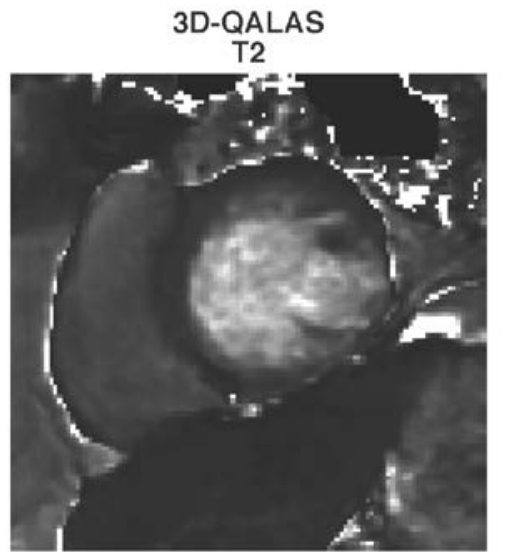

GraSE

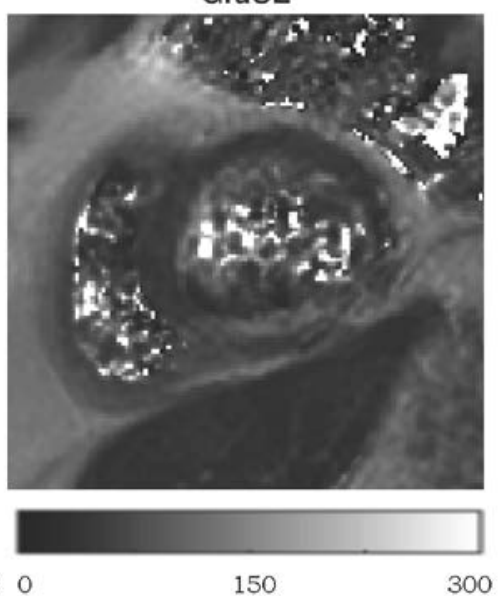


Figure 6. Native and post contrast T1 maps and T2 maps with 3D-QALAS and the reference methods from a patient with ischemic cardiomyopathy.

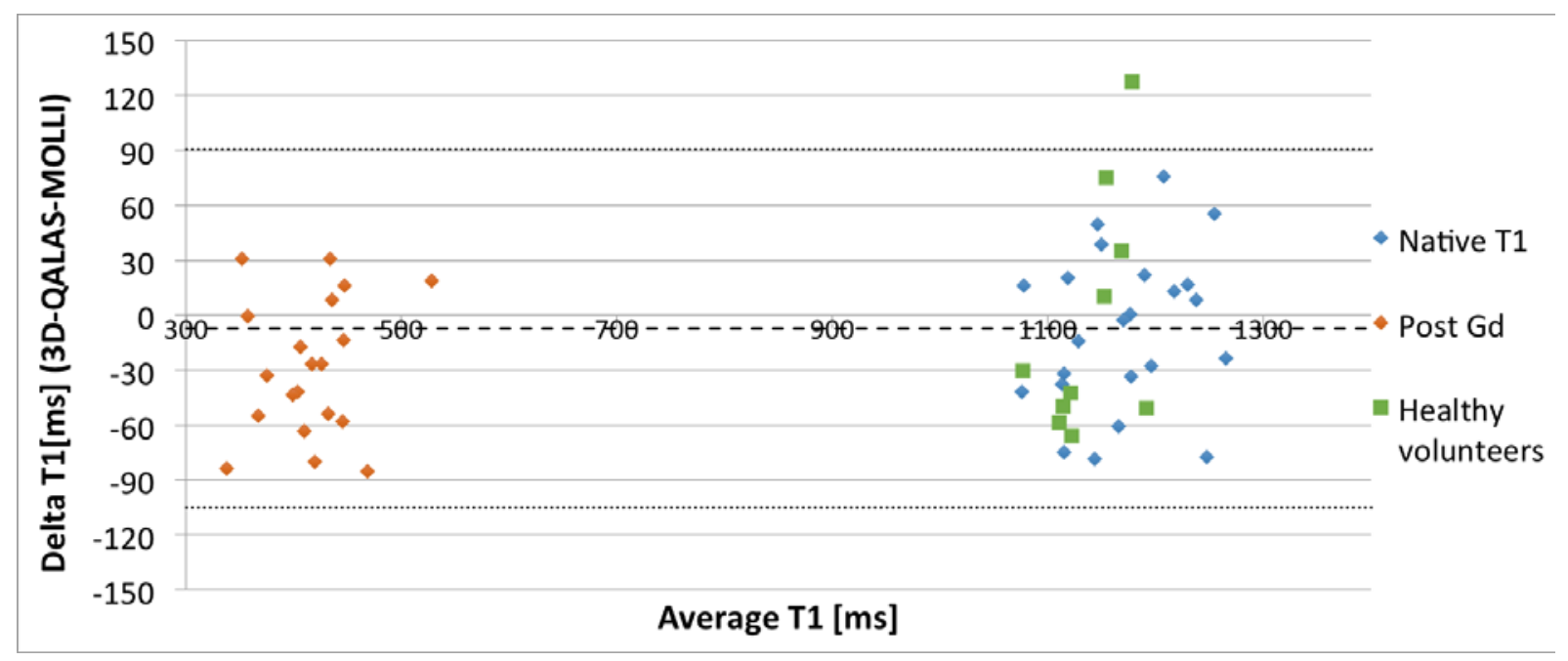

Figure 7. Bland-Altman plot for native and post contrast myocardial T1 relaxation times from 23 patients and 10 healthy volunteers. Dashed lines represent bias and $\pm 1.96 \mathrm{SD}$.

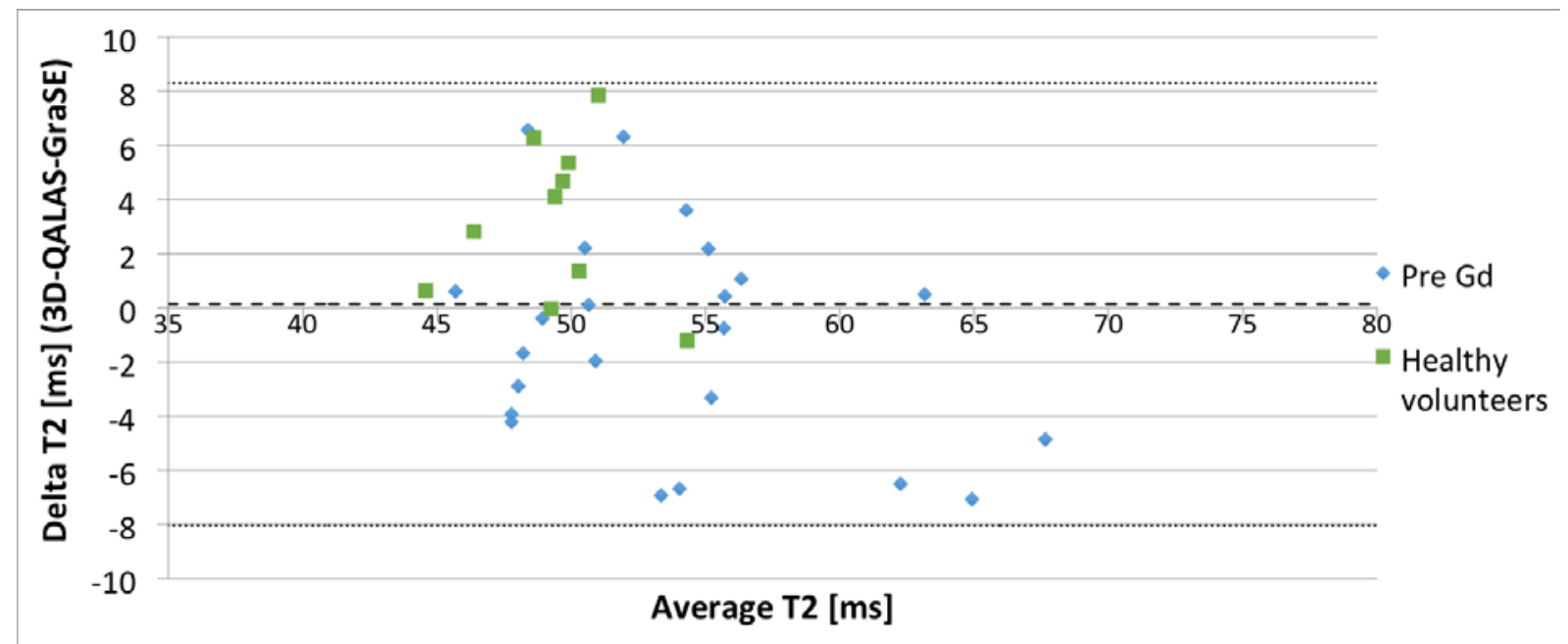

Figure 8. Bland-Altman plot for native myocardial T2 relaxation times from 23 patients and 10 healthy volunteers. Dashed lines represent bias and \pm 1.96 SD. 